\title{
Dual-Frequency Defect-Mode Lasing in Aperiodic Distributed Feedback (ADFB) Cavities
}

DOI:

10.1109/LPT.2016.2561079

Document Version

Accepted author manuscript

Link to publication record in Manchester Research Explorer

\section{Citation for published version (APA):}

Folland, T., \& Chakraborty, S. (2016). Dual-Frequency Defect-Mode Lasing in Aperiodic Distributed Feedback (ADFB) Cavities. IEEE Photonics Technology Letters, 28(15), 1617-1620.

https://doi.org/10.1109/LPT.2016.2561079

\section{Published in:}

IEEE Photonics Technology Letters

\section{Citing this paper}

Please note that where the full-text provided on Manchester Research Explorer is the Author Accepted Manuscript or Proof version this may differ from the final Published version. If citing, it is advised that you check and use the publisher's definitive version.

\section{General rights}

Copyright and moral rights for the publications made accessible in the Research Explorer are retained by the authors and/or other copyright owners and it is a condition of accessing publications that users recognise and abide by the legal requirements associated with these rights.

\section{Takedown policy}

If you believe that this document breaches copyright please refer to the University of Manchester's Takedown Procedures [http://man.ac.uk/04Y6Bo] or contact uml.scholarlycommunications@manchester.ac.uk providing relevant details, so we can investigate your claim.

\section{OPEN ACCESS}




\title{
Dual-Frequency Defect-Mode Lasing in Aperiodic Distributed Feedback (ADFB) Cavities
}

\author{
Thomas G. Folland and Subhasish Chakraborty
}

\begin{abstract}
In this letter we aim to provide the first in-depth study of a multiband hologram filter response under the influence of gain. We find that these aperiodic DFB gratings, which are essentially computer optimized digital holograms, can be inversedesigned to possess multiple defect-like modes analogous to those present in quarter-wave phase shifted gratings. We attribute this phenomenon to the interaction between multiple photonic band gaps in aperiodic DFB cavities. Further examination of the aperiodic DFB gratings reveal that the defect-mode lasing solutions are insensitive to variations of the grating feedback strength $(\kappa)$. This result in particular, which has important significance to minimize error in fabrication, is confirmed against published experimental data for an aperiodic DFB laser.
\end{abstract}

Index Terms-Lasers, Distributed Feedback Lasers, Laser Resonators, Semiconductor Lasers.

\section{INTRODUCTION}

$\mathbf{C}$ ONTROLLING the color of light emitted by a laser requires careful design of the resonant cavity used. Although the traditional target for resonator design is single frequency lasing, reliable multicolor emission is also appealing for other applications. For example, coherent frequency generation requires the photomixing of two lasers in a nonlinear medium to generate spectrally pure light at a sum or difference frequency [1]. By generating both frequencies in the same cavity the common-noise effect will significantly reduce the linewidth of generated light, thereby relaxing phase stability requirements on the laser. Distributed feedback (DFB) gratings are one of the most conventional ways of achieving frequency selective mode control. The conventional uniform DFB grating reflects light at twice the grating period $(\Lambda)$ through Bragg scattering, leading to a photonic stop-band at the Bragg wavelength $\left(\lambda_{\mathrm{B}}=2 \Lambda n_{\mathrm{eff}}\right.$, where $n_{\mathrm{eff}}$ is the grating effective index) [2], [3]. Distributed feedback lasers based on uniform gratings show a mode spectrum with two degenerate modes with identical threshold gain values at the stop band edges [4], which may result in dual color emission. The spacing between the two band-edge lasing modes and their corresponding threshold values are intrinsically linked through the grating feedback strength $(\kappa)$, so it is difficult to control the mode spacing without affecting laser performance. Furthermore small perturbations to the DFB cavity, such as non-zero

Manuscript received Feburary 17, 2016; revised April 14, 2016; accepted April XX, XXXX. Date of publication May XX, XXXX; date of current version June XX, XXXX. TGF acknowledges the North-West Nanoscience Doctoral Training Centre, EPSRC grant EP/G03737X/1

T. Folland and S. Chakraborty are with the School of Electrical and Electronic Engineering, The University of Manchester, Manchester M13 9PL, U.K. (e-mail: thomas.folland@manchester.ac.uk; s.chakraborty@manchester.ac.uk;).

Digital Object Identifier 10.1109/LPT.XXXX.XXXXXX

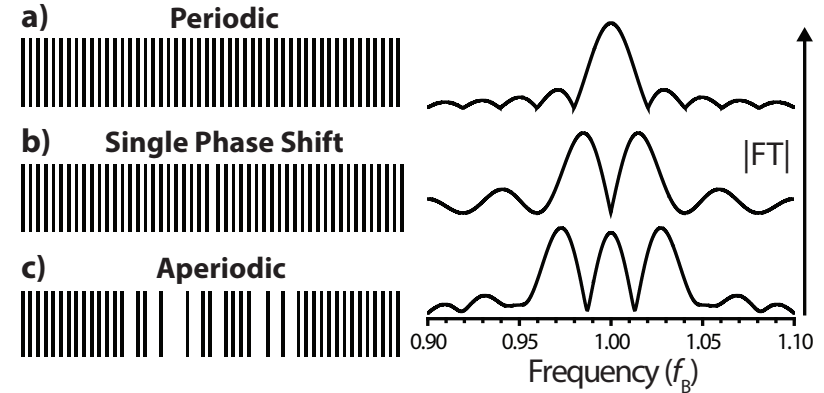

Fig. 1. Illustration of spatial domain holograms a) - c) show holograms with an increasing number of spatial frequency components, represented by a uniform grating, a grating with a quarter-wave phase-shift and an aperiodic lattice.

facet reflectivity, can break the threshold degeneracy between band-edge modes [4], leading to single mode emission.

A common way to induce robust single frequency emission from a DFB laser is to break the real-space uniformity by introducing a quarter-wave phase-shift or defect into the structure; this leads to a defect-mode within the photonic stop band at $\lambda_{\mathrm{B}}$. However, with a single quarter-wave phase-shift, designers are restricted to only one defect-mode. Relaxing the grating spatial uniformity further allows almost any desired spectral response to be achieved, resulting in the formation of an aperiodic lattice [5]. The recently developed discretely tunable aperiodic DFB (ADFB) laser is a strikingly successful example of such an approach [6]-[8], in which multiple defects are incorporated into a grating using aperiodic lattice engineering to simultaneously enable precise frequency selection and tuning. Unlike other approaches [9]-[12], the aperiodic lattice design is not the output of a deterministic mathematical operation; rather it is a computer-optimized digital hologram. The hologram contains a multitude of phase-shifts, the precise locations and sizes of which are set such that they operate collectively to provide a well-defined set of spatial frequency components, as revealed by the Fourier transform (FT) of the hologram [5], [6]. To illustrate the physical structure of such aperiodic lattices, in Fig. 1 we present uniform, quarter-wave shifted and ADFB gratings of the same length (see methodology for synthesis). Each grating has a distinct FT response, with one, two and three spatial frequency components for uniform, quarter-wave shifted and ADFB gratings respectively, as indicated on the right-hand side of Fig. 1.

The key advantage of the ADFB technology, to define and control resonances at multiple wavevectors with high resolution in the first Brillouin zone, has been discussed in detail elsewhere [5], [6]. The ADFB grating plays two roles: 
(i) it provides mode selection photonic bands at user-defined frequencies, and (ii) it enables electronic switching between these bands when integrated within a Fabry-Perot (FP) laser cavity with a current-controllable spectral gain shape. The second point has been much discussed in our prior work [8], where we employed time-domain modeling to calculate the lasing mode spectra of the compound system (i.e. the combined FP and grating). However, a detailed calculation of the ADFB filter response in the presence of gain was not performed, and as such the grating FT (Fig. 1) does not directly reveal the frequency and threshold of lasing modes. In this paper we study the multiband ADFB (i.e. digital hologram) filter response under the influence of gain. The methodology, which exploits the transfer matrix method (TMM) to calculate the ADFB reflectivity and group delay filter functions, is similar to techniques described in references [12]-[14]. We show that the ADFB gain-reflection response contains infiniteamplification (finite output for zero input) singularities at which the laser oscillation condition is satisfied. At these singularities the device enters a slow light regime which can result, for example, in dramatic increase of light-matter interaction within a gain medium [3]. The ADFB singularities can be designed to behave like defect-modes, the frequencies of which can be directly inferred from the grating FT (unlike band edge modes). Indeed, the introduction of gain to the ADFB filter function has been instrumental in enabling us to predict modal amplification within a graphene controlled laser [15].

\section{Methodology}

The aperiodic lattice (Fig1) is inverse designed by setting a target filter function $\tilde{\kappa}(f)$, [8] and using a binary search algorithm with simulated annealing (SA) to optimize the lattice; here $\tilde{\kappa}(f)$ contains two high transmission defect states within a wide photonic stop band [5]. The SA algorithm starts by initially selecting a random grating structure to form a trial lattice. The spectral response of the trial lattice is calculated using the FT method described elsewhere [5], [6], [8], giving an initial spectral response. The difference between the target spectral characteristics and the actual spectral response(i.e, the FT of the trial lattice), describing the 'error', is then calculated and used as the cost function for the SA optimization process. On each subsequent iteration, the algorithm modifies a randomly selected grating element, generating a new trial grating and re-computing the cost function. The new trial grating is then evaluated in an annealed, probabilistic fashion. Specifically, if the recomputed cost function falls below a predetermined cost function value then the trial grating is accepted and the process continues for further optimization. To ensure that the search process avoids becoming trapped in any local minima at the early stages of the optimization process, small increases in the cost function are also accepted according to Maxwell-Boltzmann classical probability statistics. As the system 'cools' the probability of accepting positive increase in cost function reduces, and the system tends towards a global minimum in cost space, producing an optimal grating design.

To calculate the forward problem (i.e. the filter response of a hologram under the influence of gain) accurately we exploit a
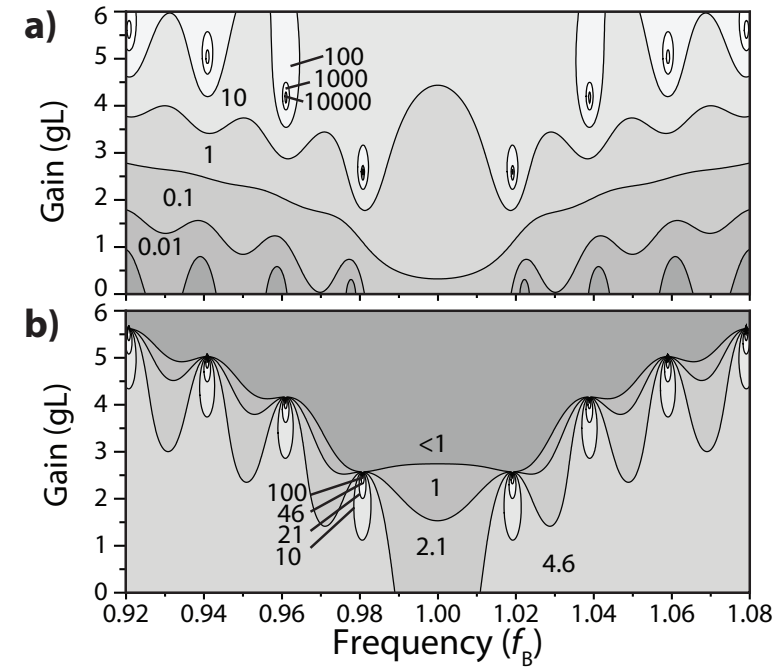

Fig. 2. Calculated a) reflectivity and b) group delay (ps) response functions for a periodic grating for $\kappa L=1.5$. The results are in agreement with coupled wave theory.

TMM based on [12], [16]. In brief, the forward and backward propagating waves are related through a system transfer matrix M. This matrix can be expressed as the product of a series of sub-matrices $\left(\mathbf{T}_{i}\right)$, governing the propagation and scattering within grating element with an index of $n_{1}$ or $n_{2}$. Scattering was calculated using the Fresnel coefficients for reflection at the boundaries between layers [16] and normalized to the grating coupling constant $\kappa L$, where $n_{2}-n_{1}=\Delta n=\kappa \lambda_{\mathrm{B}} / 2$ and $L$ is the grating length. To remove the difference in optical path length between grating elements of $n_{1}$ and $n_{2}$ propagation was performed according to the geometrically averaged effective index, $n_{\text {eff }}$. Material gain $(g)$ is included in simulations by adding an imaginary component to $n_{\text {eff }}$. We can express the reflection and transmission coefficients from the components $\left(T_{i j}\right)$ of the matrix $\mathbf{M} ; r=T_{12} / T_{11}$ and $t=1 / T_{11}$ (and corresponding power reflectivity and transmission $R=|r|^{2}, T=|t|^{2}$ ). To calculate the spectral group delay $\tau(\omega)$ of a wave propagating through the structure we utilize the following expression in terms of the transmission phase $\phi_{t}[17]$;

$$
\tau(\omega)=\frac{d \phi_{t}}{d \omega}
$$

As group delay is intrinsically linked to the electromagnetic density of modes (DOM) [3], [18], we can use it to study the photonic band structure properties of ADFB gratings. To analyze the properties of lasing modes and the DOM we calculate the reflection coefficient and group delay filter response over the full frequency-gain $(f, g)$ plane. These results can be represented by a contour plot, where the reflectivity or group delay is represented by contour information as a variable of $f$ (normalized to $f_{\mathrm{B}}=c / \lambda_{\mathrm{B}}$ ) and $g$. The condition for selfoscillation is provided by the points where $R(f, g) \rightarrow \infty$, corresponding to a finite output for zero input.

\section{RESUlTS AND DISCUSSION}

In Fig. 2 we present the properties of the uniform grating, characterized by a single dominant Fourier component, in 


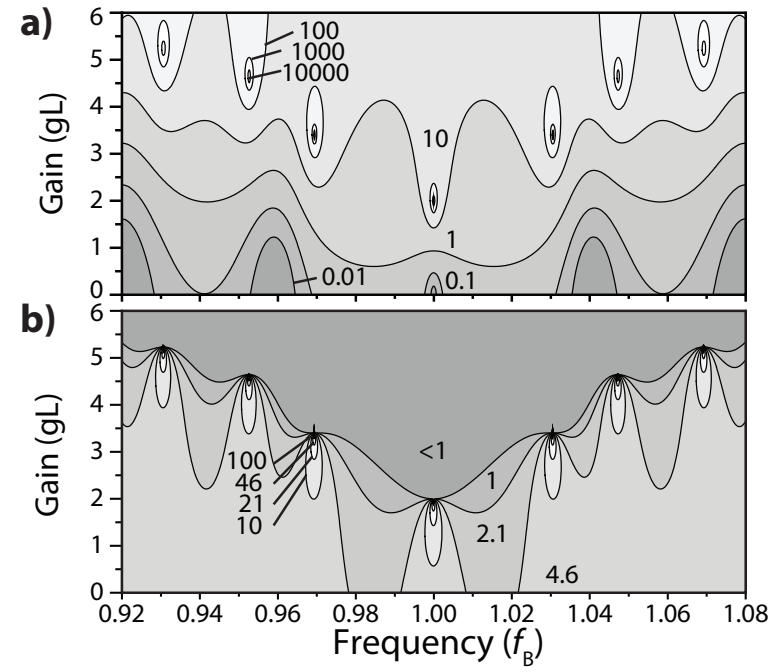

Fig. 3. Calculated a) reflectivity and b) group delay (ps) response functions for a single quarter-wave defect grating for $\kappa L=1.5$

terms of both reflectivity and group delay (or DOM). The behavior of uniform gratings in general are well known, but are discussed here to provide a more systematic understanding of singularities in the gain-reflectivity and gain-group delay response functions. The plots show a series of singularities in both response functions, which represent the frequency and gain values at which self-oscillation occurs. Furthermore, we note that a single reflection band, as provided by a uniform grating, contributes two degenerate singularities with identical threshold gain values at the band-edges to the filter response. This is the band-edge enhancement effect as discussed at length in earlier work, indicated here by the peaks in the group delay response function [3], [14]. We can now study these properties in ADFB structures.

Reflectivity and group delay results are presented for a single quarter-wave defect, characterized by two symmetric Fourier components each contributing a single reflectivity band around $f_{\mathrm{B}}$, in Fig. 3. As discussed above, each reflection band should contribute two singularities to the filter function at the band edges. However, as two reflection bands are placed sufficiently close together, specifically at the two immediately resolvable Fourier-points around $f_{\mathrm{B}}$ [5], [6], two singularities will coincide and the group delay (or DOM) is enhanced by both band-edges [14]. This leads to a strong resonance at $f_{\mathrm{B}}$ with decreased threshold compared with the uniform grating [14], which can be viewed as a defect state in a broad photonic bandgap. We also note the two singularities at the opposite edge of each Bragg band at significantly higher gain values, which are conventional band-edge states as observed in the uniform grating. We finally discuss the aperiodic lattice (characterized by three dominant Fourier components as shown in Fig. 1(c)); the results are shown in Fig. 4. Once again each Fourier component contributes a single reflectivity band and then each band will contribute two singularities to the filter function. In this case due to the choice of the Fourier points we find that bands overlap at two frequencies on either side of $f_{\mathrm{B}}$. As in the single-defect structure, the DOM combine

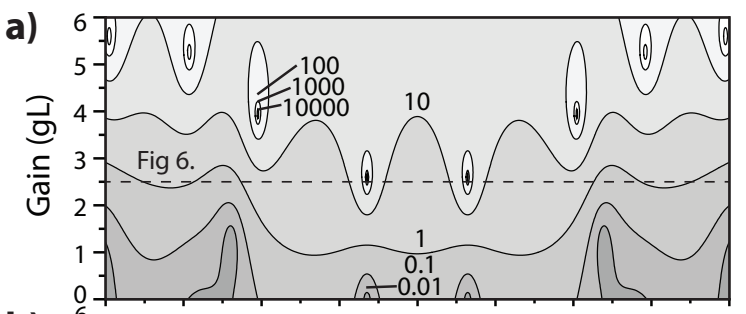

b)

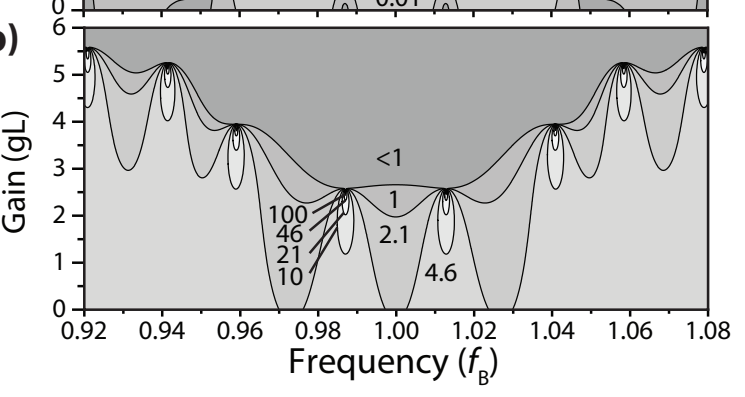

Fig. 4. Calculated a) reflectivity and b) group delay (ps) response functions for an aperiodic grating for $\kappa L=1.5)$. The dotted line represents data plotted in Fig. 6

at each of these points, leading to two singularities of lower threshold than the outer band-edge modes.

Now that we have addressed the existence of the singularities in the ADFB grating response, we can assess the robustness of the modes in these structures. To do this we examine the positions of the reflectivity and group delay maxima as the grating coupling strength is changed (shown for all three structures in Fig. 5). For the uniform grating as the coupling strength is increased, the band edge modes display continuous tuning. This is due to the broadening of the photonic band gap for a stronger coupling strength [2]. However, for the case of the single-defect and multi-defect ADFB gratings the lowest threshold defect modes show no such frequency movement as the grating coupling strength is varied. Note that the higher threshold modes still change frequency suggesting they act as conventional band edge states. This suggests that the two principal modes in the multidefect ADFB grating are of similar nature to that of the wellknown quarter-wave single defect grating.

The insensitivity of the ADFB defect mode singularities to coupling strength has important experimental consequences. To this end, in Fig. 6 we compare results plotted in Fig. 4 ( $\kappa L=1.5, g L=2.5$ ) with the dominant laser modes (red dots) presented in reference [19], where an ADFB grating following the design in Fig. 1(c) has been integrated into a terahertz Quantum Cascade Laser [6]-[8]. In contrast to [19], where laser emission was explained qualitatively using the peak position of the ADFB reflectivity bands within the limited gain bandwidth, here we match the measured lasing mode positions to defect-mode singularities predicted by the TMM. To do this, first we exploit the global symmetry of the ADFB response function (see Fig. 4 and $5 \mathrm{c}$ ) across $f_{\mathrm{B}}$. Thereafter, the only fitting parameter required for matching is the Bragg frequency $\left(f_{\mathrm{B}}=4.325 \mathrm{THz}\right)$, defined by the grating period $\Lambda(=9.51 \mu \mathrm{m}$ for this device) and the effective index $n_{\mathrm{eff}}(=3.644$, fitted). Therefore, if the gain medium has sufficient bandwidth to cover both modes, we can control grating parameters $\Lambda$ and 

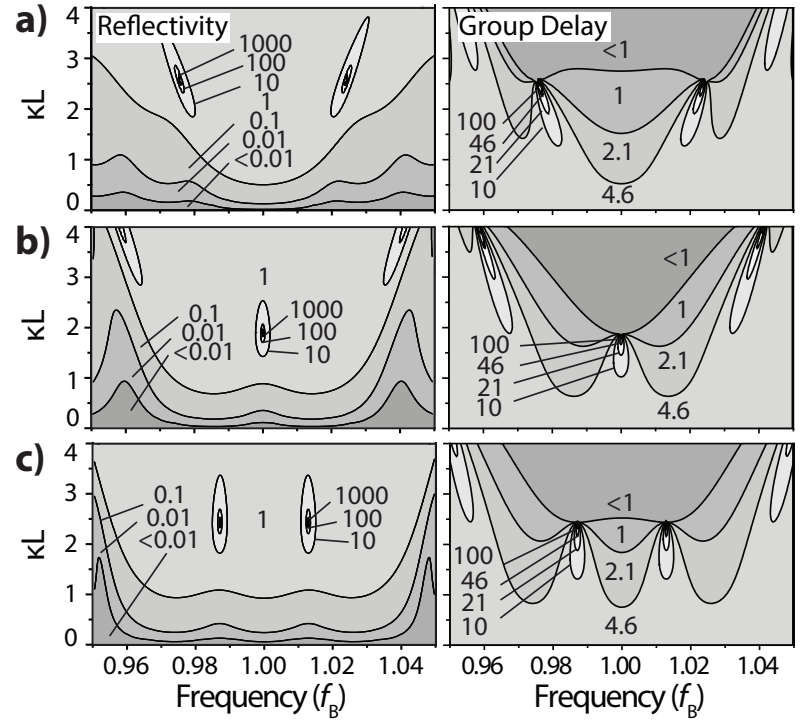

Fig. 5. Reflectivity and group delay (ps) response functions in the $(f, \kappa L)$ plane, for gratings with $g L=1.5$; a) uniform, b) quarter-wave phase-shift and c) ADFB.

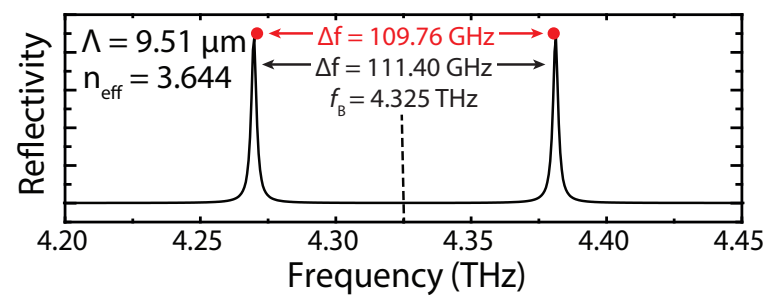

Fig. 6. Comparison between reflectivity calculated in this paper and dominant laser emission frequencies (represented as red dots) presented in [19]. Each mode dominates at a different operational current. Discrete mode tuning and the small observable deviation between theory and experiment is attributed to feedback from FP facets.

$n_{\text {eff }}$ to place $f_{\mathrm{B}}$ at the laser gain centre wavelength and meet the design specification for achieving defect-mode lasing at predetermined frequencies. The remarkable similarity between experimentally measured and numerically calculated results support this conclusion. However, the presence of FP facets is a potential source of error in fabrication (further to $\Lambda$ and $n_{\text {eff }}$ ), as the frequency spacing between modes can be sensitive to the random location of cleaved facets. Indeed, there is a small observable deviation between simulation (mode spacing $111.40 \mathrm{GHz}$ ) and experiment (mode spacing $109.76 \mathrm{GHz}$ ). This $1.6 \mathrm{GHz}$ deviation in mode positions can be attributed to FP facet reflection, which is competing with the grating feedback and thereby perturbs the ADFB mode solutions [4].

\section{CONCLUSIONS}

In this letter we have discussed the response function of holographically designed multi-defect aperiodic DFB gratings under the influence of gain. The ADFB gratings are characterized by a well-defined set of Fourier components; we have hypothesized that each of these components will contribute two band-edge modes to the grating response. Depending on the positioning of these modes they may combine, leading to a robust low threshold defect state which does not vary in frequency with grating feedback strength. By using numerically accurate transfer matrix techniques to study these gratings we find that the results agree with our hypothesis. We have then shown that these results can be used to predict the dominant mode frequencies of a $\mathrm{THz}$ semiconductor laser with an embedded ADFB grating. These results suggest that hologram design techniques could be used to produce gratings with a large number of closely spaced defect resonances. As well as previously demonstrated applications of discrete electronic tuning, these structures could see applications in difference frequency generation.

\section{REFERENCES}

[1] M. Tani et al., "Generation of coherent terahertz radiation by photomixing of dual-mode lasers," Optical and Quantum Electronics, vol. 32, no. 4-5, pp. 503-520, 2000.

[2] A. Yariv and P. Yeh, Photonics: Optical Electronics in Modern Communications. Oxford University, 2007.

[3] J. P. Dowling, M. Scalora, M. J. Bloemer, and C. M. Bowden, "The photonic band edge laser: A new approach to gain enhancement," Journal of Applied Physics, vol. 75, no. 4, p. 1896, Feb. 1994.

[4] L. A. Coldren, S. W. Corzine, and M. L. Mashanovitch, Diode Lasers and Photonic Integrated Circuits. John Wiley \& Sons, 2012.

[5] S. Chakraborty, M. C. Parker, and R. J. Mears, "A Fourier (k-) space design approach for controllable photonic band and localization states in aperiodic lattices." Photonics and Nanostructures - Fundamentals and Applications., vol. 3, no. 2-3, pp. 139-147, Dec. 2005.

[6] S. Chakraborty et al., "Longitudinal computer-generated holograms for digital frequency control in electronically tunable terahertz lasers," Applied Physics Letters, vol. 101, no. 12, p. 121103, 2012.

[7] O. P. Marshall et al., "Electronically tunable aperiodic distributed feedback terahertz lasers," Journal of Applied Physics, vol. 113, no. 20, p. 203103, 2013.

[8] S. Chakraborty et al., "Discrete mode tuning in terahertz quantum cascade lasers." Optics Express, vol. 20, no. 26, pp. B306-14, Dec. 2012.

[9] V. Jayaraman, Z.-M. Chuang, and L. Coldren, "Theory, design, and performance of extended tuning range semiconductor lasers with sampled gratings," IEEE Journal of Quantum Electronics, vol. 29, no. 6, pp. 1824-1834, Jun. 1993.

[10] I. Avrutsky et al., "Design of widely tunable semiconductor lasers and the concept of binary superimposed gratings (BSG's)," IEEE Journal of Quantum Electronics, vol. 34, no. 4, pp. 729-741, Apr. 1998.

[11] L. Mahler et al., "Quasi-periodic distributed feedback laser," Nature Photonics, vol. 4, no. 3, pp. 165-169, Jan. 2010.

[12] G. Adolfsson, J. Bengtsson, and A. Larsson, "Spectral engineering of semiconductor Fabry-Perot laser cavities in the weakly and strongly perturbed regimes," Journal of the Optical Society of America B, vol. 27, no. 1, p. 118, Dec. 2009.

[13] G. F. Barlow and K. A. Shore, "Application of the argument principle method to the calculation of DFB laser diode modes," International Journal of Numerical Modelling: Electronic Networks, Devices and Fields, vol. 14, no. v, pp. 291-302, 2001.

[14] A. G. Smirnov, D. V. Ushakov, and V. K. Kononenko, "Multiplewavelength lasing in one-dimensional bandgap structures: implementation with active nipi layers," Journal of the Optical Society of America B, vol. 19, no. 9, p. 2208, 2002.

[15] S. Chakraborty et al., "Gain modulation by graphene plasmons in aperiodic lattice lasers," Science, vol. 351, no. 6270, pp. 246-248, Jan. 2016.

[16] M. C. Troparevsky, A. S. Sabau, A. R. Lupini, and Z. Zhang, "Transfermatrix formalism for the calculation of optical response in multilayer systems: from coherent to incoherent interference." Optics express, vol. 18 , no. 24 , pp. 24715-21, Nov. 2010.

[17] A. Papoulis, The Fourier Integral and its Applications, 1st ed. Mc Graw-Hill, 1962.

[18] J. Bendickson, J. Dowling, and M. Scalora, "Analytic expressions for the electromagnetic mode density in finite, one-dimensional, photonic band-gap structures," Physical Review E, vol. 53, no. 4, pp. 4107-4121, Apr. 1996.

[19] S. Chakraborty et al., "Mode Switching using Weak Aperiodic DFB Gratings within Fabry-Pérot Lasers," in PECS-X, 2012, pp. 340-341. 